\title{
VP6-SUMO Self-Assembly as Nanocarriers for Gastrointestinal Delivery
}

\author{
V. Palmieri, ${ }^{1}$ F. Bugli, ${ }^{2}$ M. Papi, ${ }^{1}$ G. Ciasca, ${ }^{1}$ G. Maulucci, ${ }^{1}$ S. Galgano, ${ }^{2}$ A. Arcovito, ${ }^{3}$ \\ M. Sanguinetti, ${ }^{2}$ and M. De Spirito ${ }^{1}$ \\ ${ }^{1}$ Istituto di Fisica, Università Cattolica del SC, L. Go F. Vito 1, 00168 Roma, Italy \\ ${ }^{2}$ Istituto di Microbiologia, Università Cattolica del SC, L. Go F. Vito 1, 00168 Roma, Italy \\ ${ }^{3}$ Istituto di Biochimica e Biochimica Clinica, Università Cattolica del SC, L. Go F. Vito 1, 00168 Roma, Italy \\ Correspondence should be addressed to M. Papi; m.papi@rm.unicatt.it
}

Received 8 September 2015; Revised 5 November 2015; Accepted 8 November 2015

Academic Editor: Andrea Falqui

Copyright (C) 2015 V. Palmieri et al. This is an open access article distributed under the Creative Commons Attribution License, which permits unrestricted use, distribution, and reproduction in any medium, provided the original work is properly cited.

\begin{abstract}
High proteolytic degradation and poor absorption through epithelial barriers are major challenges to successful oral delivery of therapeutics. Nanoparticle platforms can enhance drug stability and extend the residence time in gastrointestinal (GI) tract. However, drug delivery systems are often inactivated in acidic environment of stomach or suffer poor absorption from intestinal cells due to the mucus layer. To overcome these issues we developed a drug delivery system constituted by a protein construct made by a Rotavirus capsid protein (VP6) and the small ubiquitin-like modifier SUMO. This chimeric construct allows specificity towards intestinal cells, the Rotavirus natural target, combined by an enhanced stability given by the eukaryotic protein transporter SUMO. Furthermore SUMO can act as a molecular switch that facilitates import/export of its ligand to the nucleus, the hypersensitive subcellular site target of many cell killing therapies. In this paper we show that SUMO-VP6 constructs self-assembly into stable nanocarriers. SUMO-VP6 nanocarriers display ideal features for drug delivery: a small size and high monodispersity, a high stability in different $\mathrm{pH}$ conditions and a high uptake in the nuclear and cytoplasmic compartment of intestinal cells. These features make SUMO-VP6 nanocarriers a promising novel system for oral delivery of poorly soluble drugs.
\end{abstract}

\section{Introduction}

In the field of drug delivery there is a continuous effort for optimization of pharmaceutical formulation to obtain a maximal accumulation in the targeted tissue $[1,2]$. Oral delivery is the most widely used form of drug administration for compounds directed toward the gastrointestinal (GI) tract.

Despite the advantage of the high absorptivity of intestinal epithelium, oral formulations face several problems, such as (i) poor stability in the gastric environment, (ii) low solubility and/or bioavailability, and (iii) low penetration in the mucus barrier, which prevents drug absorption [3].

To overcome these limitations and design a carrier with high specificity towards intestinal cells combined to stability in GI environment, we produced a construct made of VP6 protein fused with the Small Ubiquitin Like Modifier (SUMO). VP6 protein is the most immunogenic, antigenic and abundant protein of the capsid of Rotavirus, the prominent causative agents of gastroenteritis in children [4, 5]. VP6 protein, whose natural target is represented by intestinal cells, can assemble in structured scaffolds, such as trimers, spheres, and tubes having different immunogenic and delivery properties. These structures have received considerable attention for the development of gastroenteritis vaccines and for GI delivery of therapeutics [5-7]. Though the targeting and modulation of the geometry of particles should give the possibility to control the pharmacokinetics and intestinal cell endocytosis [1], in vivo studies with VP6 nanoparticles have demonstrated a loss of the protein after intragastric administration due to intraluminal degradation of VP6 [5]. In order to increase stability in vivo in the GI environment, in our formulation, VP6 has been conjugated with SUMO. SUMO is ubiquitously expressed in eukaryotic cells where it is reversibly conjugated to specific targets to modulate 
their localization, interactions, and stability $[8,9]$. For this reason, SUMO is used in intermediate steps of biotechnology protocols to enhance the solubility of proteins expressed and purified from E. coli [7]. Moreover, SUMO conjugation can induce nuclear uptake and release, since SUMO regulates nuclear transport and is removed from targets by specific nucleoplasmic proteases [10].

In this paper, we demonstrate that the expression of SUMO-VP6 constructs allows the production of a uniform population of nanoassemblies with controlled size and shape. These nanoassemblies are monodisperse and stable at $\mathrm{pH}$ values characteristic of GI tract. Moreover, SUMO-VP6 carriers have the capacity of entering intestinal cell lines and reach the cell nucleus, a fundamental process in the development of drug delivery systems designed for GI tract diseases.

\section{Materials and Methods}

2.1. Expression of VP6 and VP6-SUMO Fusion Protein. VP6 and VP6-SUMO proteins were produced as previously reported [7]. Briefly, the VP6-coding region from human rotavirus RNA was cloned into a pET-SUMO expression vector using the TA cloning technique (Invitrogen). The $E$. coli strains Top-10 and BL21 (DE3) (Thermo Fisher Scientific) were used as host cells for subcloning and VP6 expression, respectively. After purification from bacteria lysate by using a nickel column with Ni-NTA resin, proteins were analysed in $10 \%$ sodium dodecyl sulfate polyacrylamide gel electrophoresis (SDS-PAGE), and the expression yield was analysed with Nanodrop Spectrophotometer (Thermo Scientific). For VP6 production the SUMO-VP6 protein was reacted with SUMO protease (Genecopoeia) at $30^{\circ} \mathrm{C}$ for $6 \mathrm{~h}$. The cleaved SUMOVP6 samples were applied to a new nickel column to obtain the purified VP6 by subtracting the $6 \mathrm{x}$ His-tagged proteins (SUMO protease and SUMO-VP6). The purified proteins were checked by SDS-PAGE, and the samples were stored at $-80^{\circ} \mathrm{C}$ for further assay.

\subsection{Dynamic Light Scattering (DLS) and 3D Structure Simu-} lations. Dynamic light-scattering measurements on protein solutions were carried out with a Zetasizer Nano S (Malvern Instruments, Malvern, UK) equipped with a $4 \mathrm{~mW} \mathrm{He}-\mathrm{Ne}$ laser $(633 \mathrm{~nm})$. Measurements were performed at $37^{\circ} \mathrm{C}$ at an angle of $173^{\circ} \mathrm{C}$ from the incident beam. Peak-intensity analyses were used to determine hydrodynamic radius by number distribution $(P(n))$ in different buffer conditions as described elsewhere [11]. Theoretical 3D models of SUMOVP6 were generated by using i-Tasser [12-14]. Hydrodynamic properties of 3D structures were calculated as described elsewhere $[15,16]$ with the software Hydropro [17].

2.3. Small Angle X-Ray Scattering (SAXS). SAXS data were collected at the BM29 beamline at the European Synchrotron Radiation Facility (ESRF) of Grenoble. Data were analysed as explained elsewhere $[15,18]$. Briefly, for accurate subtraction of the scattering deriving from cell and solvent, buffer scattering was measured. Two-dimensional SAXS patterns were azimuthally averaged in order to obtain the scattered intensity
$I(q)$ as a function of the X-ray-exchanged momentum $(q)$ in the range from 0.05 to $4.27 \mathrm{~nm}^{-1}$. Data were converted to absolute intensity values by normalising to the scattering signal of water. Each acquisition was performed for $1 \mathrm{~s}$ and then averaged in order to avoid sample damage, with measurement successively repeated at different positions on each sample to augment the number of statistics for analysis. Measurements were performed at $37^{\circ} \mathrm{C}$ at a concentration of $1 \mathrm{mg} / \mathrm{mL}$. Software package ATSAS 2.4 was used for data analysis as described elsewhere [19]. Briefly, after buffer signal subtraction, global structural parameters were extracted from data by using program PRIMUS. Particle size was estimated from the radius of gyration $\left(R_{G}\right)$ by using the Guinier approximation. Estimation of the maximum particle diameter was obtained through the pair-distance distribution function $P(r)$ calculated by the indirect Fourier transform program GNOM.

2.4. Transmission Electron Microscopy (TEM). Samples for transmission electron microscopy were dropped on carboncoated glow-discharged copper grids. The samples were allowed to adsorb on the carbon film for 5 minutes, and sample excess was removed with filter paper. After staining with a $2 \%$ uranyl acetate solution for 30 seconds in the dark at room temperature, staining excess was removed with filter paper and the grid washed with ultrapure water. Grids were air-dried before observation at room temperature. TEM micrographs were collected with a Libra 120 (Carl Zeiss, Germany).

2.5. Cell Culture and Spinning Disk Confocal Microscopy. The HT29 human colon carcinoma-derived cell line was purchased from the American Type Culture Collection (ATCC; Manassas, VA) and maintained in DMEM as previously reported [11]. Uptake and intracellular distribution of SUMOVP6 nanoassemblies in HT29 cells were investigated by means of immunofluorescence assay as previously reported [20]. Briefly, when cells reached confluence, samples were washed three times with PBS. Then, $100 \mu \mathrm{L}$ of culture medium containing $20 \mu \mathrm{L}$ of VP6-SUMO $(1 \mathrm{mg} / \mathrm{mL})$ was added and incubated at $37^{\circ} \mathrm{C}$. At fixed time points $(2 \mathrm{~h}$ and $24 \mathrm{~h})$, cells were washed with PBS three times and fixed with precooled methanol for $20 \mathrm{~min}$. The cells were dehydrated with ethanol solutions, washed with PBS, and then treated with $0.3 \%$ Triton X-100 (Sigma Aldrich). Then anti-HIS antibody (1:500 in PBS, Qiagen) was added to the cells and incubated at $37^{\circ} \mathrm{C}$ for $30 \mathrm{~min}$. The unbound antibodies were removed by washing with PBS-Tween (0.2\%). Afterward, FITC-labeled anti-IgG antibody (Qiagen) 1:400 in a solution 1:4 of PBS and Evans Blue were added and incubated at $37^{\circ} \mathrm{C}$ for $30 \mathrm{~min}$. Finally, the cells were washed five times with PBS-Tween. Cell nuclei were stained with Fluoromount G with DAPI (Electron Microscopy Sciences) as reported previously [21].

Immunofluorescence images were obtained with a multichannel white light source with DAPI/FITC/Evans Blue filter settings on a CARV II spinning-disk microscope (Crisel Instruments, Rome, Italy) by using a $60 \mathrm{x}$ oil immersion objective (NA 1.4). $Z$-stacks were acquired for each sample. 


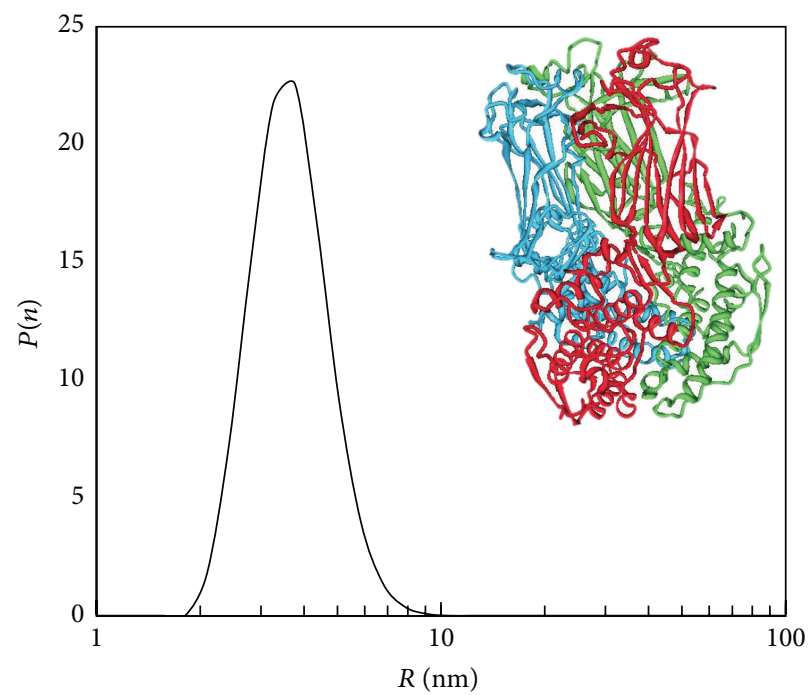

FIGURE 1: Number weighted distribution of VP6 and crystallographic structure of VP6 trimer (PDB: 1QHD). By means of Hydro- pro software a theoretical value of $3.4 \mathrm{~nm}$ has been calculated for the VP6 trimer, consistent with the DLS mean hydrodynamic radius of $3.8 \mathrm{~nm}$. In the insert the $\mathrm{PDB}$ of $3 \mathrm{D}$ structure of the trimer is shown.

Background values (defined as intensities below $7 \%$ of the maximum intensity) were set to zero and coloured black. Image processing and analysis was performed using ImageJ software on $Z$-stacks. Cell boundaries have been determined by the Evans Blue emission in each $Z$ plane. Particles outside boundaries have been removed from each plane and excluded from analysis. Nuclear region in each plane was determined via DAPI channel. Particles inside the nucleus were counted and then subtracted from the images. After this subtraction, the $Z$-projection for each cell has been calculated and the number of particles per cell was calculated by using "Analysing Particle tool" of ImageJ.

\section{Results and Discussion}

Drug delivery systems for GI tract must face the barrier of the viscous, elastic, and sticky mucus layer, which rapidly traps and removes foreign particles [22]. Mucus size filtering allows particles that are smaller than the cut-off size of the hydrogel to pass, whereas it rejects larger objects. Since nanoparticle uptake is size-dependent, VP6 and SUMO-VP6 size and monodispersity have been carefully evaluated.

VP6 protein from human Rotavirus A has been expressed in an E. coli BL21 system following a recently developed protocol [7]. Purified protein at $\mathrm{pH} \mathrm{7,4} \mathrm{in} \mathrm{Tris-HCl} \mathrm{buffer}$ self-assembles into trimers which are known to be the thermodynamically most stable form of VP6 under many conditions $[23,24]$. A representative VP6 number weighted distribution $(P(n))$ is shown in Figure 1. VP6 displays a mean hydrodynamic radius $\left(R_{H}\right)$ of $3.8 \mathrm{~nm}$. This radius is similar to the theoretical value of $3.4 \mathrm{~nm}$ obtained with Hydropro from the crystallographic structure of the trimeric assembly of VP6 (PDB 1QHD shown in the inset of Figure 1).

If the SUMO-VP6 construct is expressed, the trimeric form is lost and the chimeric protein self-assembles in a new thermodynamically stable structure. Size properties of this SUMO-VP6 assembly have been studied by means of Dynamic Light Scattering (DLS), Small Angle X-Ray Scattering (SAXS), and Transmission Electron Microscopy (TEM).

SUMO-VP6 at $\mathrm{pH} 7.4$ in Tris- $\mathrm{HCl}$ buffer has a $R_{H}$ of $7.8 \mathrm{~nm}$ as is visible from the $P(n)$ distribution in Figure 2(a). The protein has a narrow size distribution indicating a high monodispersity. SAXS has been performed to retrieve radius of gyration $\left(R_{G}\right)$ and pair distribution function $P(R)$ of the protein. The $P(R)$ distribution is bell-shaped indicating a compact globular structure with a $R_{G}$ of $7.9 \mathrm{~nm}$, consistent with value obtained with DLS. This value has been confirmed also with Guinier analysis.

High resolution Transmission Electron Microscopy has been used to characterize shape features of the nanocarriers. Representative image in Figure 2(c) shows the monodisperse small globular structures formed by SUMO-VP6. From images a mean radius of $11 \pm 2 \mathrm{~nm}$ has been calculated. By means of i-Tasser software the $3 \mathrm{D}$ structure of a single SUMO-VP6 construct has been simulated and the best model has been used to calculate theoretical hydrodynamic properties. The theoretical $R_{H}$ value from the $3 \mathrm{D}$ structure is $3.3 \mathrm{~nm}$, much smaller than the experimental $R_{H}$ of $7.9 \mathrm{~nm}$; hence we can conclude that several subunits self-assemble to form a single carrier. Stability of SUMO-VP6 nanoassemblies in solution has been evaluated with DLS after $24 \mathrm{~h}$ (data not shown) and no significative variations of $R_{H}$ have been measured showing that these structures are highly stable.

To verify the stability of nanocarriers at the different $\mathrm{pH}$ encountered in the GI tract, the nanoparticles have been dialyzed against acetate buffer at $\mathrm{pH} 2$ to simulate stomach environment, against MOPS at $\mathrm{pH}$ 6,5 in order to simulate oral $\mathrm{pH}$ and Tris- $\mathrm{HCl}(\mathrm{pH} 7,4)$ to simulate small intestine $\mathrm{pH}$. In Figure 3 the $R_{H}$ of SUMO-VP6 assemblies at $\mathrm{pH} 2$, $\mathrm{pH} 6.5$, and $\mathrm{pH} 7,4$ are compared. The assemblies display a high stability and monodispersity even at extremely low $\mathrm{pH}$. 


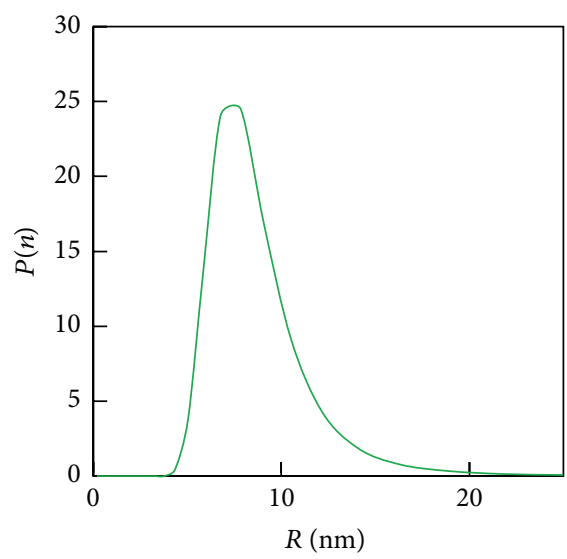

(a)

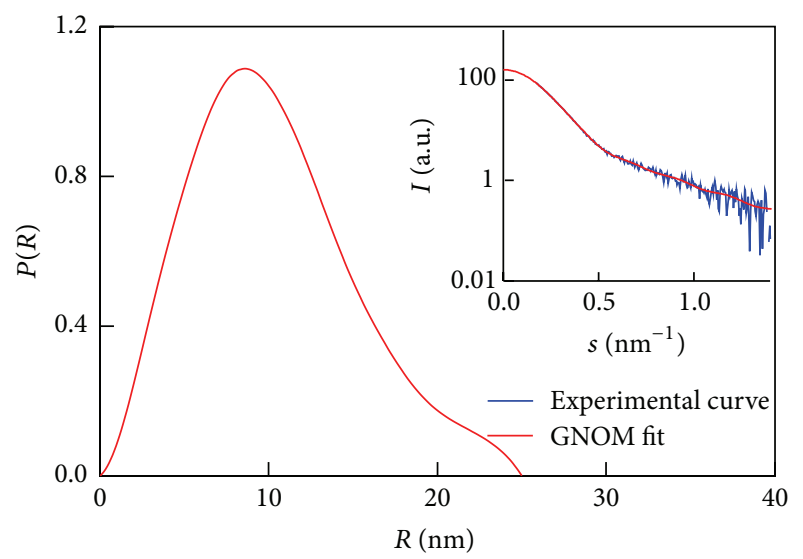

(b)

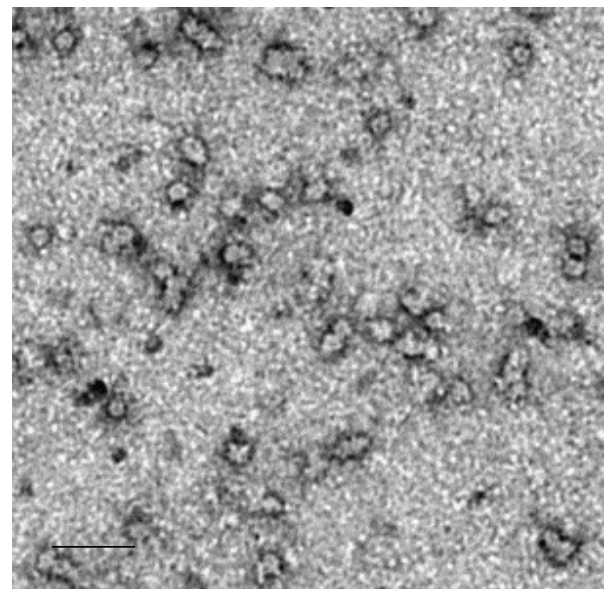

(c)

FIgure 2: (a) $P(n)$ distribution of SUMO-VP6 oligomer obtained with DLS (b) $P(r)$ distribution of VP6-Sumo oligomer from SAXS measurement. A representative SAXS curve is shown in the inset in blue. The fit performed by GNOM is in red in the inset. In (c), a representative TEM image of SUMO-VP6 nanoassemblies, scale bar is $100 \mathrm{~nm}$.

In Figure 3 three representative size distributions are displayed showing the high consistency of the $P(n)$ distributions at different $\mathrm{pH}$. No significative changes in size have been measured after $24 \mathrm{~h}$ at all $\mathrm{pH}$ studied (data not shown).

To explore the capacity of SUMO-VP6 nanocarriers to deliver exogenous proteins to intestinal cells, interaction experiments were performed between nanocarriers and cultured HT29 colon cancer cells. Confocal Z-stacks were acquired for each cell in order to distinguish between assemblies in nuclear compartment (labelled with DAPI) or extranuclear compartment.

$Z$ projections of cells labelled with Evans blue (green) and viral assemblies (labelled with FITC and shown in red in the image) are shown in Figure 4. Multiple particles per cell are visible with a uniform distribution in the whole cell after $2 \mathrm{~h}$ of treatment (Figures 4(a) and 4(b) with zoom on a representative cell in (c-d)) and in the cytoplasm after $24 \mathrm{~h}$ of treatment (Figures $4(\mathrm{e})$ and $4(\mathrm{~d})$ with zoom on a representative cell in $(\mathrm{g}-\mathrm{h}))$.

Analysis of confocal images showed that the nanoassemblies are distributed uniformly in the whole cell with a high uptake and an average of 26 particles per cell distributed half in the cytoplasm and membranes half in the nucleus after $2 \mathrm{~h}$ of treatment (Figure 5). The SUMO-VP6 nanoassemblies represent a suitable biodegradable carrier for nuclear compartment.

Since from DLS measurements we know that particle is stable in solution after $24 \mathrm{~h}$, we evaluated the stability in cell compartments at the same time point. After $24 \mathrm{~h}$ of treatment the number of particles up taken in the nuclear compartment significantly decreases to an average of 2.4 particles/cell (Figure 5).

\section{Conclusions}

Oral delivery remains the preferred route of drug administration due to its noninvasive nature and improved patient compliance [3]. With advances in molecular biology and biotechnology, the repertoire of therapeutic compounds has extended beyond small molecular weight compounds to include proteins, peptides, large biological macromolecules, supramolecular structures, nanoparticles, and even whole 

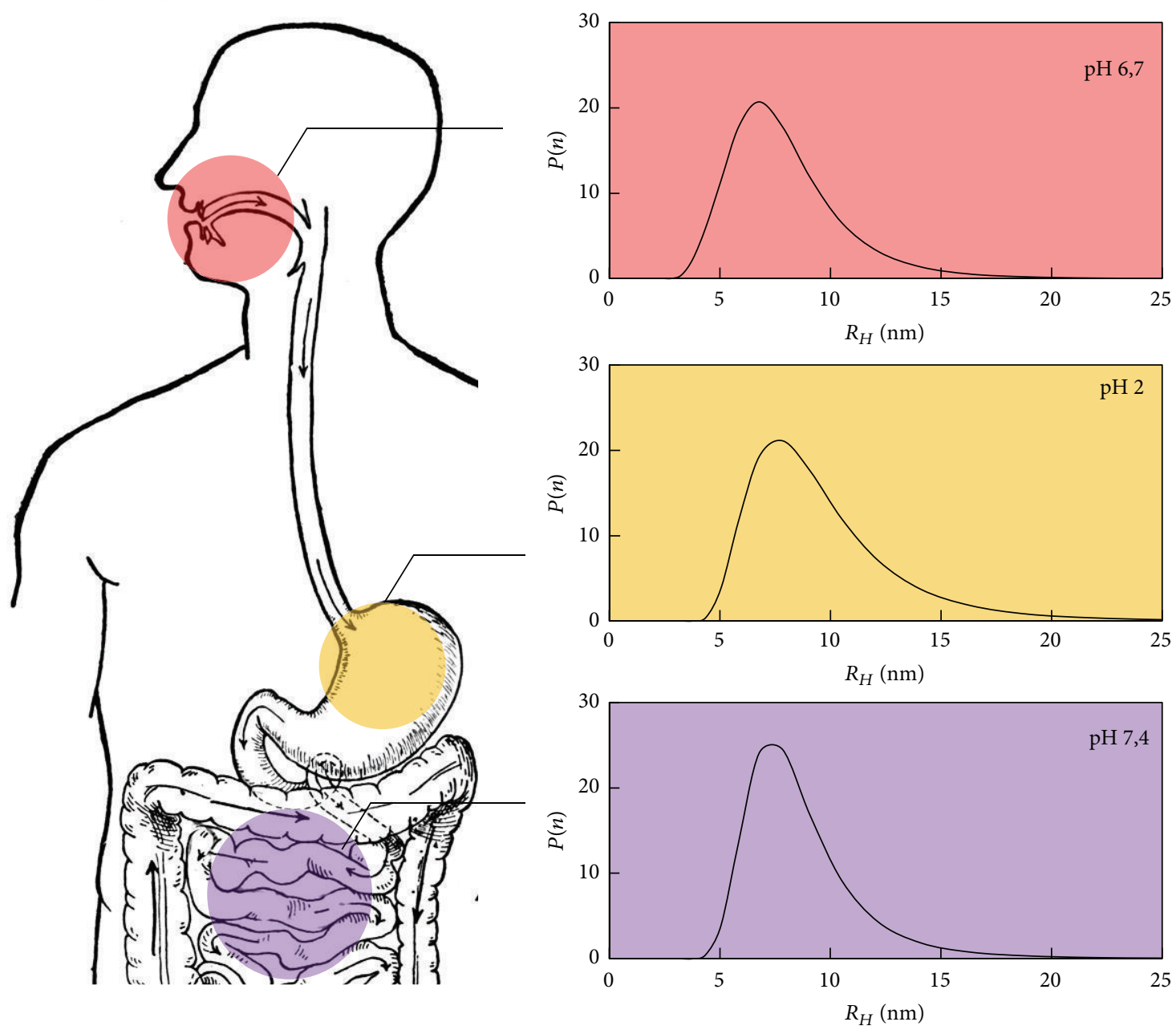

FIGURE 3: SUMO-VP6 nanoassemblies stability at different $\mathrm{pH}$. The $\mathrm{pH}$ of mouth, stomach, and small intestine has been used to evaluate the nanocarriers stability. Three representative $P(n)$ distributions of particles at different $\mathrm{pH}$ are shown. The radius of the particle is stable around $8 \mathrm{~nm}$ going from $\mathrm{pH} 2$ (yellow) to $\mathrm{pH} 6,7$ (red) and $\mathrm{pH} 7,4$ (purple).

cells [22]. However, many of these biological therapeutics have poor oral bioavailability due to formidable barriers posed by gastrointestinal tract and are therefore rarely used for oral drug delivery. The typical physiology of GI tract leads to poor bioavailability due to several factors including low mucosal permeability and/or degradation of a drug before absorption. Oral administration of such compounds with improved bioavailability requires effective drug delivery systems before successful use in humans. Therapeutic agents such as proteins, DNA, or RNA are highly soluble but poorly permeable and are generally the most susceptible to degradation in the GI tract. The transport of larger compounds, such as nanoparticles, can be restricted by the layer of mucus that lines the GI tract. Furthermore, the epithelial lining of the intestine has poor nonspecific permeability for most water-soluble macromolecules. Finally the transport of small molecular weight compounds is limited by poor mucosal or biofilm permeability and solubility [23-25].

To overcome these limitations we engineered a chimeric construct combining the Rotavirus capsid protein VP6 for its specificity towards intestinal cells with the eukaryotic protein transporter SUMO to enhance the stability, the cellular uptake, and the delivery to the cellular nucleus.

We showed that this protein construct is able to selfassemble producing very stable particles of $\sim 20 \mathrm{~nm}$ of diameter. This novel nanoparticle, for its high stability at different $\mathrm{pH}$ and for its little size able to cross the intestinal mucus filtering, is a promising biotechnological platform to transport pharmacological compounds through the GI tract [2].

Finally we showed that these nanoparticles are uptaken in HT29 colon cancer cells and reach the nuclear compartment. This makes this delivery system particularly suitable for release of pharmacological compounds in the nucleus, primary site of action of many pharmaceutical drugs.

Better understanding of the routes of drug absorption (paracellular and transcellular), proteolytic enzyme susceptibility, stability, and degradation of macromolecules during the in vivo transport will allow the development and an effective optimization of this technology for gastrointestinal drug delivery. 


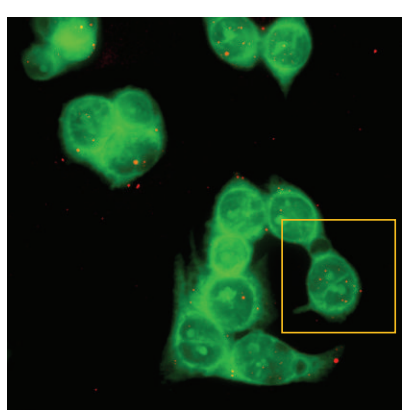

(a)

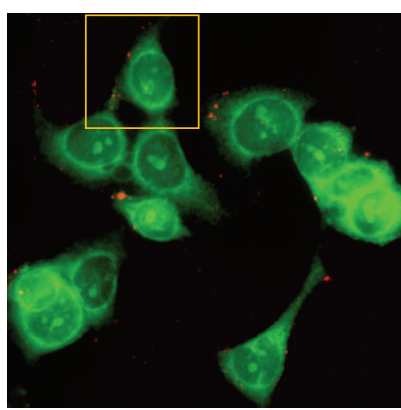

(e)

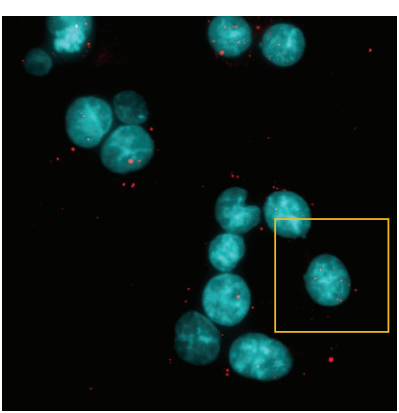

(b)

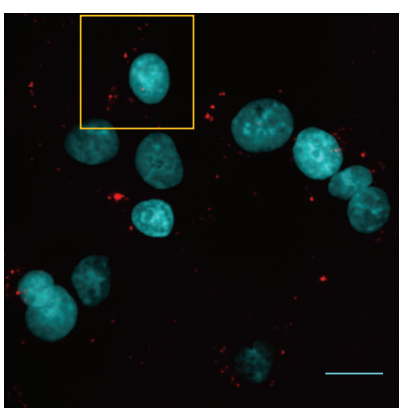

(f)

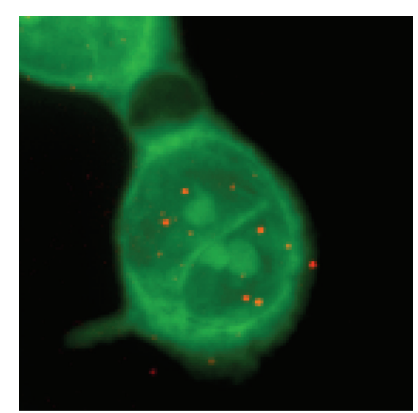

(c)

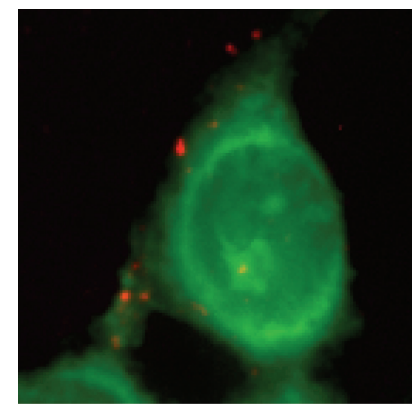

(g)

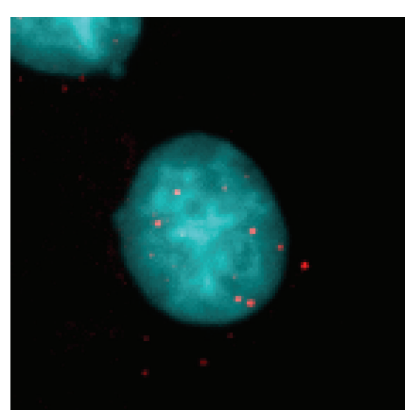

(d)

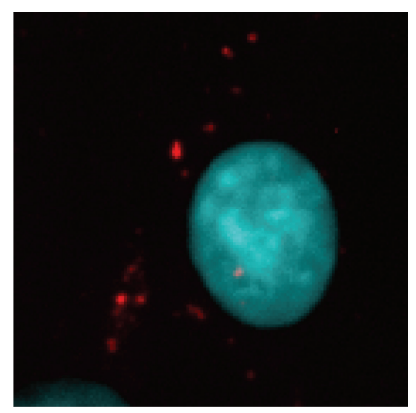

(h)

FIGURE 4: Confocal microscopy of VP6-SUMO uptake in HT29 colon cancer cells. Nanoparticles highlighted in red and superimposed with whole cell in green (after $2 \mathrm{~h}$ of treatment in $(\mathrm{a}-\mathrm{c})$ and after $24 \mathrm{~h}$ of treatment in $(\mathrm{e}-\mathrm{g})$ ) or nucleus in cyan (after $2 \mathrm{~h}$ of treatment in (b-d) and after $24 \mathrm{~h}$ of treatment in (f-h)). Scale bar in figure is $20 \mu \mathrm{m}$.

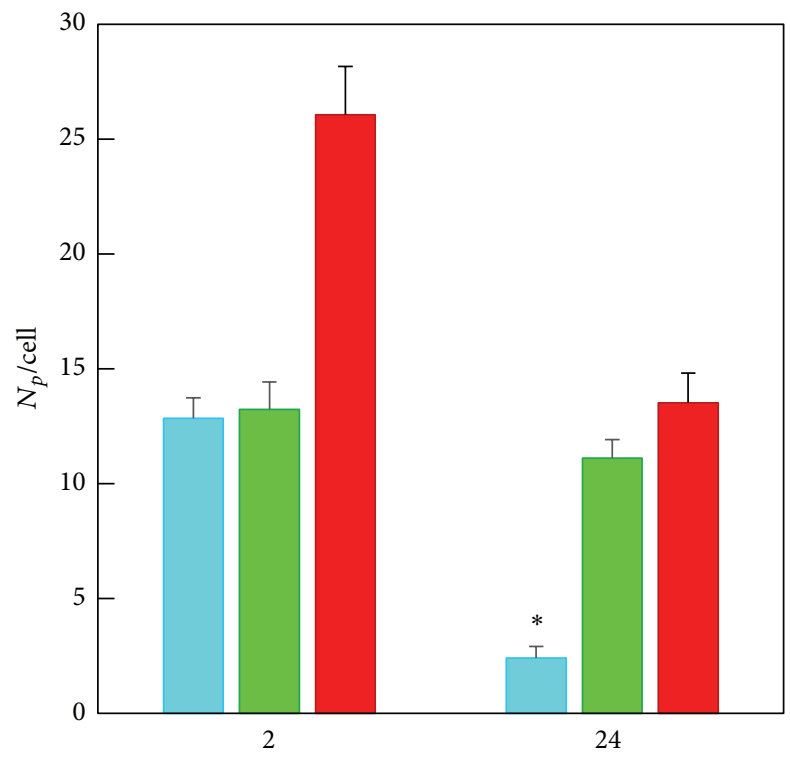

(h)

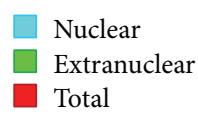

Figure 5: Number of particles per cell has been calculated and compared after $2 \mathrm{~h}$ or $24 \mathrm{~h}$ of cell treatment with SUMO-VP6 nanocarriers. While nanoparticle number remains stable after $24 \mathrm{~h}$ of treatment in extranuclear compartment, the nanoparticles number in the nucleus is significatively reduced. 50 cells per sample in 3 separated experiments have been analysed. Significance has been evaluated by ANOVA test and is indicated by an asterisk in the graph.

\section{Conflict of Interests}

The authors declare that there is no conflict of interests regarding the publication of this paper.

\section{Authors' Contribution}

V. Palmieri and F. Bugli equally contributed to this work.

\section{Acknowledgments}

This research has been supported by Università Cattolica del Sacro Cuore of Rome. SAXS measurements were performed at the BM29 beamline at the European Synchrotron Radiation Facility (ESRF), Grenoble, France. Measurements were performed at the Laboratorio Centralizzato di Microscopia Ottica ed Elettronica facility (LABCEMI) of Università Cattolica del S. Cuore (Rome, Italy). The authors are extremely thankful to Mario Amici for the technical support in experiments.

\section{References}

[1] A. Albanese, P. S. Tang, and W. C. W. Chan, “The effect of nanoparticle size, shape, and surface chemistry on biological systems," Annual Review of Biomedical Engineering, vol. 14, pp.1-16, 2012.

[2] J. Renukuntla, A. D. Vadlapudi, A. Patel, S. H. S. Boddu, and A. K. Mitra, "Approaches for enhancing oral bioavailability of peptides and proteins," International Journal of Pharmaceutics, vol. 447, no. 1-2, pp. 75-93, 2013. 
[3] L. M. Ensign, R. Cone, and J. Hanes, "Oral drug delivery with polymeric nanoparticles: the gastrointestinal mucus barriers," Advanced Drug Delivery Reviews, vol. 64, no. 6, pp. 557-570, 2012.

[4] S. Jalilvand, S. M. Marashi, and Z. Shoja, "Rotavirus VP6 preparations as a non-replicating vaccine candidates," Vaccine, vol. 33, no. 29, pp. 3281-3287, 2015.

[5] N. G. Cortes-Perez, C. Sapin, L. Jaffrelo et al., "Rotavirus-like particles: a novel nanocarrier for the gut," Journal of Biomedicine and Biotechnology, vol. 2010, Article ID 317545, 10 pages, 2010.

[6] J. Lepault, I. Petitpas, I. Erk et al., "Structural polymorphism of the major capsid protein of rotavirus," The EMBO Journal, vol. 20, no. 7, pp. 1498-1507, 2001.

[7] F. Bugli, V. Caprettini, M. Cacaci et al., "Synthesis and characterization of different immunogenic viral nanoconstructs from rotavirus VP6 inner capsid protein," International Journal of Nanomedicine, vol. 9, pp. 2727-2739, 2014.

[8] F. Melchior, M. Schergaut, and A. Pichler, "SUMO: ligases, isopeptidases and nuclear pores," Trends in Biochemical Sciences, vol. 28, no. 11, pp. 612-618, 2003.

[9] O. Kerscher, "SUMO junction-what's your function? New insights through SUMO-interacting motifs," EMBO Reports, vol. 8 , no. 6 , pp. 550-555, 2007.

[10] D. Mukhopadhyay and M. Dasso, "Modification in reverse: the SUMO proteases," Trends in Biochemical Sciences, vol. 32, no. 6, pp. 286-295, 2007.

[11] V. Palmieri, D. Lucchetti, I. Gatto et al., "Dynamic light scattering for the characterization and counting of extracellular vesicles: a powerful noninvasive tool," Journal of Nanoparticle Research, vol. 16, no. 9, article 2583, 2014.

[12] A. Roy, A. Kucukural, and Y. Zhang, "I-TASSER: a unified platform for automated protein structure and function prediction," Nature Protocols, vol. 5, no. 4, pp. 725-738, 2010.

[13] Y. Zhang, "I-TASSER server for protein 3D structure prediction," BMC Bioinformatics, vol. 9, article 40, 2008.

[14] J. Yang, R. Yan, A. Roy, D. Xu, J. Poisson, and Y. Zhang, "The I-TASSER Suite: protein structure and function prediction," Nature Methods, vol. 12, no. 1, pp. 7-8, 2015.

[15] V. Palmieri, G. Maulucci, A. Maiorana, M. Papi, and M. De Spirito, " $\alpha$-crystallin modulates its chaperone activity by varying the exposed surface," ChemBioChem, vol. 14, no. 17, pp. 2362-2370, 2013.

[16] M. Chiarpotto, G. Ciasca, M. Vassalli et al., "Mechanism of aluminium bio-mineralization in the apoferritin cavity," Applied Physics Letters, vol. 103, no. 8, Article ID 083701, 2013.

[17] A. Ortega, D. Amorós, and J. G. de La Torre, "Prediction of hydrodynamic and other solution properties of rigid proteins from atomic- and residue-level models," Biophysical Journal, vol. 101, no. 4, pp. 892-898, 2011.

[18] G. Ciasca, M. Papi, M. Chiarpotto et al., "Transient state kinetic investigation of ferritin iron release," Applied Physics Letters, vol. 100, no. 7, Article ID 073703, 2012.

[19] G. Ciasca, G. Campi, A. Battisti et al., "Continuous thermal collapse of the intrinsically disordered protein tau is driven by its entropic flexible domain," Langmuir, vol. 28, no. 37, pp. 13405-13410, 2012.

[20] Q. Zhao, W. Chen, Y. Chen, L. Zhang, J. Zhang, and Z. Zhang, "Self-assembled virus-like particles from rotavirus structural protein VP6 for targeted drug delivery," Bioconjugate Chemistry, vol. 22, no. 3, pp. 346-352, 2011.
[21] V. Palmieri, D. Lucchetti, A. Maiorana et al., "Mechanical and structural comparison between primary tumor and lymph node metastasis cells in colorectal cancer," Soft Matter, vol. 11, no. 28, pp. 5719-5726, 2015.

[22] M. Papi, G. Maulucci, M. De Spirito et al., "Ristocetin-induced self-aggregation of von Willebrand factor," European Biophysics Journal, vol. 39, no. 12, pp. 1597-1603, 2010.

[23] S. K. Lai, Y.-Y. Wang, and J. Hanes, "Mucus-penetrating nanoparticles for drug and gene delivery to mucosal tissues," Advanced Drug Delivery Reviews, vol. 61, no. 2, pp. 158-171, 2009.

[24] M. Mathieu, I. Petitpas, J. Navaza et al., "Atomic structure of the major capsid protein of rotavirus: implications for the architecture of the virion," The EMBO Journal, vol. 20, no. 7, pp. 1485-1497, 2001.

[25] F. Bugli, B. Posteraro, M. Papi et al., "In vitro interaction between alginate lyase and amphotericin $\mathrm{B}$ against aspergillus fumigatus biofilm determined by different methods," Antimicrobial Agents and Chemotherapy, vol. 57, no. 3, pp. 1275-1282, 2013. 

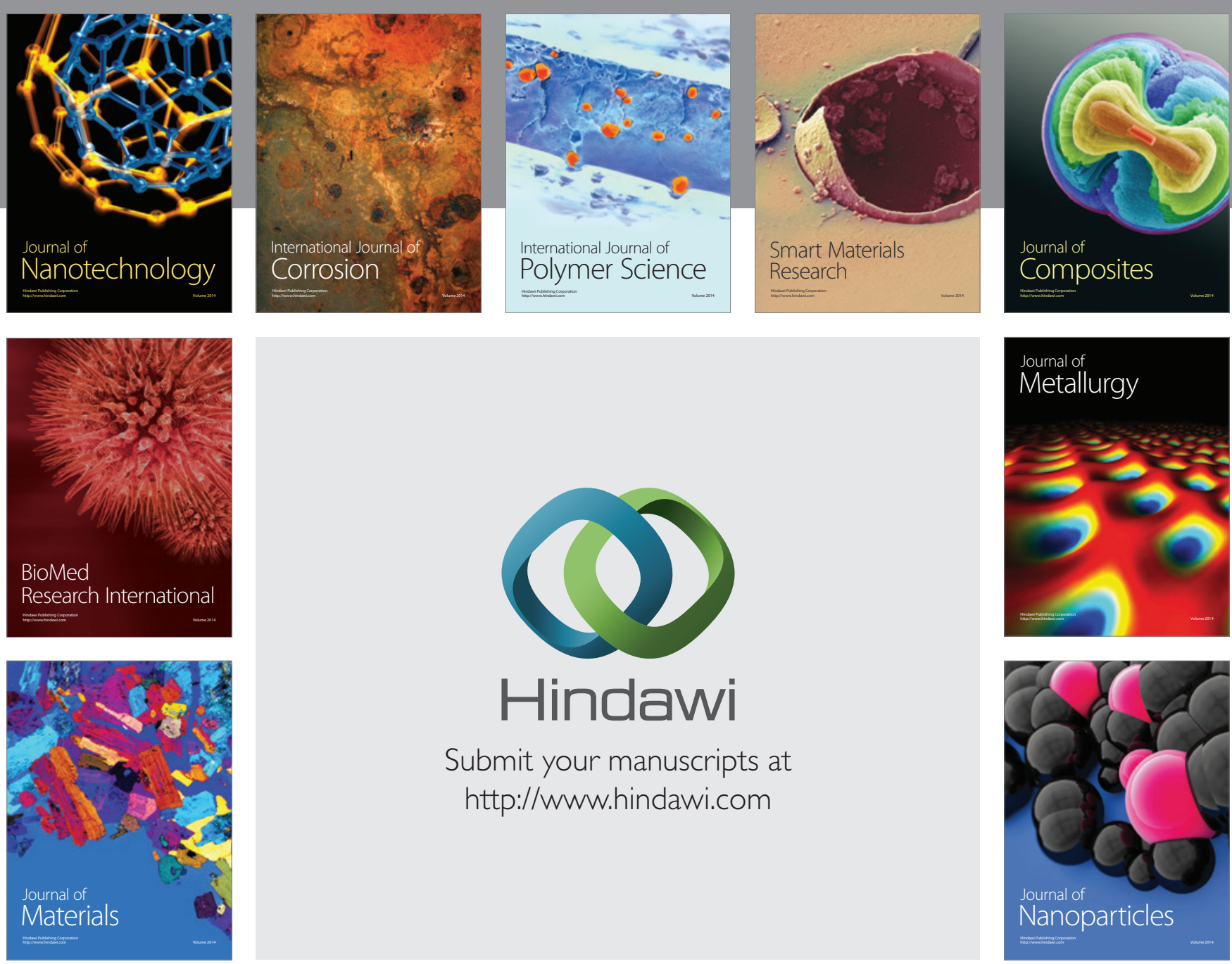

Submit your manuscripts at http://www.hindawi.com
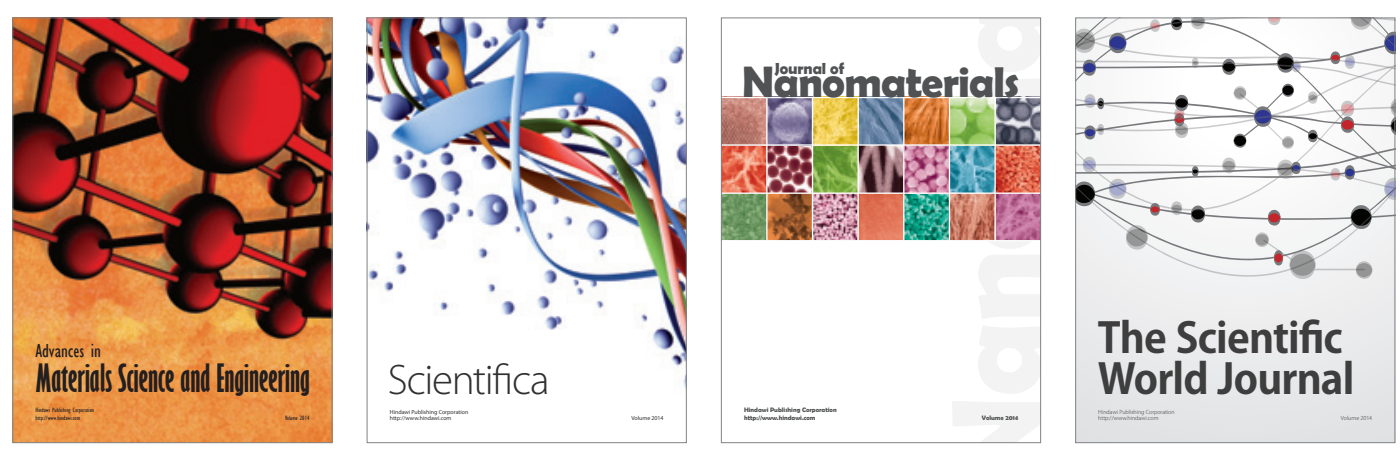

\section{The Scientific World Journal}
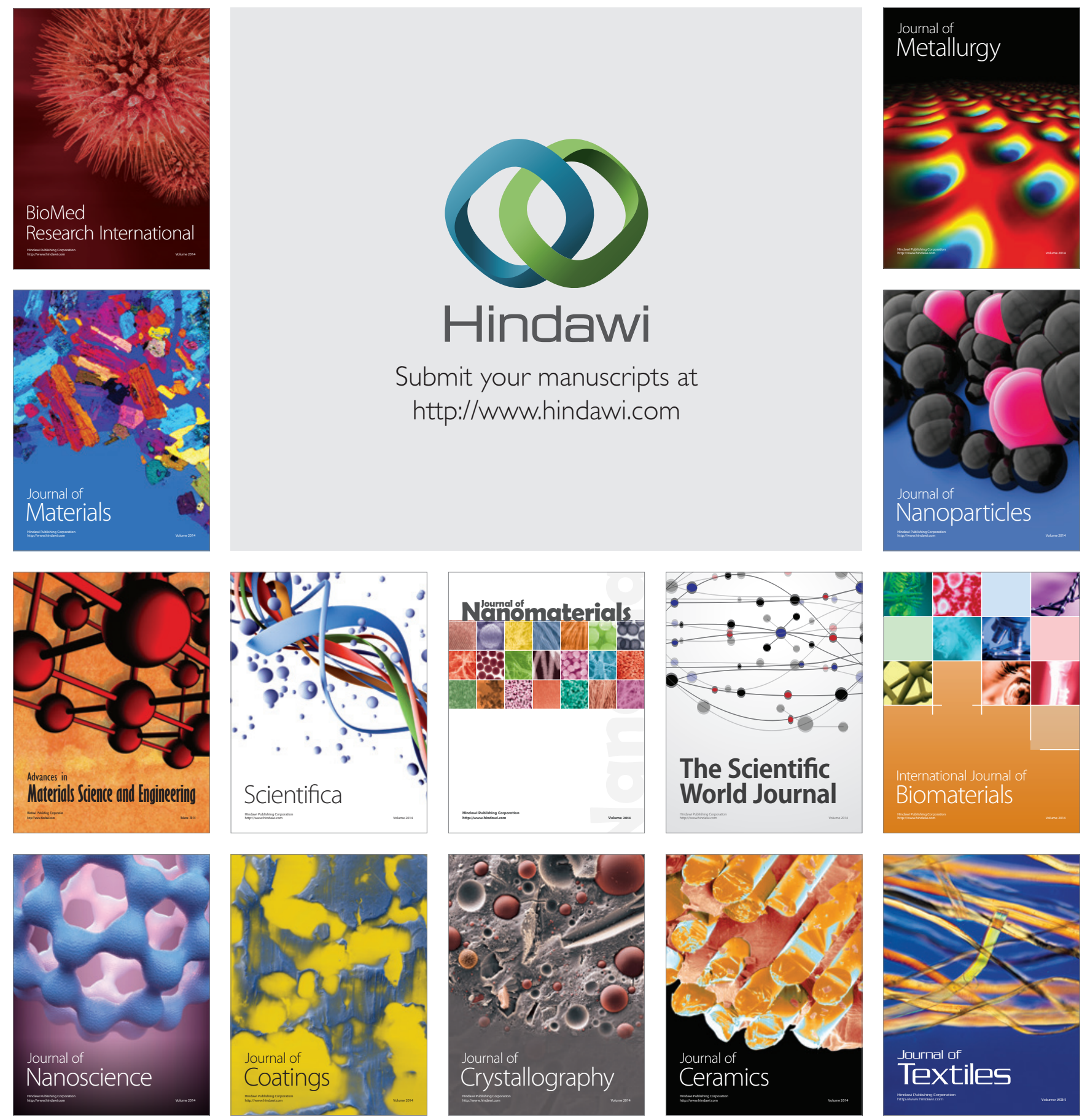